\title{
SYMMETRY OF THE MAXWELL AND MINKOWSKI EQUATIONS SYSTEM
}

\section{IVAN TSYFRA}

Communicated by Ivaïlo M. Mladenov

Abstract. We study the symmetry of Maxwell's equations for external moving media together with the additional Minkowski constitutive equations (or MaxwellMinkowski equations). We established that the system is conformally invariant.

\section{Introduction}

Symmetry properties of Maxwell equations in vacuum was studied in detail by Lorentz, Poincare, Bateman, Cuningham [1,2].

Maximal local Lie group of invariance of linear equations for electromagnetic fields in vacuum is 16 parameters group containing 15 parameter conformal group as a subgroup [3]. It was proved in [4] that the Maxwell equations in the medium, which form a system of first order partial differential equations for vectors $\vec{D}, \vec{B}$, $\vec{E}$ and $\vec{H}$, admit infinite symmetry. Thus, the system of equations

$$
\begin{array}{ll}
\frac{\partial \vec{D}}{\partial t}=\operatorname{rot} \vec{H}-\vec{j}, & \operatorname{div} \vec{D}=\rho \\
\frac{\partial \vec{B}}{\partial t}=-\operatorname{rot} \vec{E}, & \operatorname{div} \vec{B}=0
\end{array}
$$

if $\vec{j}=0, \rho=0$ is invariant under the infinite-dimensional Lie algebra with basis elements

$$
X=\xi^{\mu}(k) \frac{\partial}{\partial x_{\mu}}+\eta^{E^{a}} \frac{\partial}{\partial E^{a}}+\eta^{B^{a}} \frac{\partial}{\partial B^{a}}+\eta^{D^{a}} \frac{\partial}{\partial D^{a}}+\eta^{H^{a}} \frac{\partial}{\partial H^{a}}
$$

where

$$
\begin{aligned}
& \eta^{E_{1}}=\xi_{0}^{3} B_{2}-\xi_{0}^{2} B_{3}-\left(\xi_{1}^{1}+\xi_{0}^{0}\right) E_{1}-\xi_{1}^{2} E_{2}-\xi_{1}^{3} E_{3} \\
& \eta^{E_{2}}=-\xi_{0}^{3} B_{1}-\xi_{0}^{1} B_{3}-\left(\xi_{2}^{2}+\xi_{0}^{0}\right) E_{2}-\xi_{2}^{1} E_{1}-\xi_{2}^{3} E_{3} \\
& \eta^{E_{3}}=\xi_{0}^{2} B_{1}-\xi_{0}^{1} B_{2}-\left(\xi_{3}^{3}+\xi_{0}^{0}\right) E_{3}-\xi_{3}^{2} E_{2}-\xi_{3}^{1} E_{1}
\end{aligned}
$$




$$
\begin{aligned}
& \eta^{B_{1}}=\xi_{2}^{1} B_{2}-\xi_{3}^{1} B_{3}-\left(\xi_{2}^{2}+\xi_{3}^{3}\right) B_{1}-\xi_{3}^{0} E_{2}+\xi_{2}^{0} E_{3} \\
& \eta^{B_{2}}=\xi_{3}^{0} E_{1}-\xi_{1}^{0} E_{3}-\left(\xi_{1}^{1}+\xi_{3}^{3}\right) B_{2}-\xi_{1}^{2} B_{1}+\xi_{3}^{2} B_{3} \\
& \eta^{B_{3}}=\xi_{1}^{3} B_{1}-\xi_{2}^{3} B_{2}-\left(\xi_{1}^{1}+\xi_{2}^{2}\right) B_{3}-\xi_{2}^{0} E_{1}+\xi_{1}^{0} E_{2} \\
& \eta^{D_{1}}=\xi_{2}^{1} D_{2}+\xi_{3}^{1} D_{3}-\left(\xi_{1}^{1}+\xi_{0}^{0}\right) D_{1}+\xi_{3}^{0} H_{2}-\xi_{2}^{0} H_{3} \\
& \eta^{D_{2}}=-\xi_{3}^{0} H_{1}-\xi_{1}^{0} H_{3}-\left(\xi_{2}^{2}+\xi_{0}^{0}\right) D_{2}-\xi_{1}^{2} D_{1}+\xi_{3}^{2} D_{3} \\
& \eta^{D_{3}}=\xi_{1}^{3} D_{1}+\xi_{2}^{3} D_{2}-\left(\xi_{3}^{3}+\xi_{0}^{0}\right) D_{3}-\xi_{2}^{0} H_{1}-\xi_{1}^{0} H_{2} \\
& \eta^{H_{1}}=-\xi_{0}^{3} D_{2}+\xi_{0}^{2} D_{3}-\left(\xi_{1}^{1}+\xi_{0}^{0}\right) H_{1}-\xi_{1}^{2} H_{2}-\xi_{1}^{3} H_{3} \\
& \eta^{H_{2}}=\xi_{0}^{3} D_{1}-\xi_{0}^{1} D_{3}-\left(\xi_{2}^{2}+\xi_{0}^{0}\right) H_{2}-\xi_{2}^{1} H_{1}-\xi_{2}^{3} H_{3} \\
& \eta^{H_{3}}=\xi_{0}^{2} D_{1}-\xi_{0}^{1} D_{2}-\left(\xi_{3}^{3}+\xi_{0}^{0}\right) H_{3}-\xi_{2}^{3} H_{2}-\xi_{3}^{1} H_{1}
\end{aligned}
$$

with $\xi^{\mu}(x)$ being arbitrary smooth functions, $\xi_{\nu}^{\mu}=\frac{\partial \xi^{\mu}}{\partial x_{\nu}}, \mu, \nu=\overline{0,3}, a, b=\overline{1,3}$, and $t \equiv x_{0}$. It follows that the equations (1)-(2) are invariant with respect to any transformation of $t, \vec{x}$ forming the Lie group. At the same time the vectors $\vec{D}$, $\vec{B}, \vec{E}, \vec{H}, \vec{j}$ and the density $\rho$ are transformed on the linear representation of this group. But the system of equations (1)-(2) is undetermined. In addition to these equations we have to consider supplement constitutive equation. As was shown in [4] there are the nonlinear constitutive equation which form the Poincare and conformally invariant system of equation together with the equations (1)-(2). It contains the well known Born-Infeld nonlinear equation for electromagnetic fields as a particular case. Imposing different constraints on fields $\vec{D}, \vec{B}, \vec{E}, \vec{H}$, we obtain different constitutive equation invariant with respect to Galilei, Poincare and conformal group. More exactly, the constitutive equations

$$
\vec{D}=M \vec{E}+N \vec{B}, \quad \vec{H}=M \vec{B}-N \vec{E}
$$

where $M$ and $N$ are arbitrary functions of $I_{1}=\vec{B}^{2}-\vec{E}^{2}, I_{2}=\vec{B} \cdot \vec{E}$, are Poincare invariant [4,5] (see also [6]). If on the other hand $M \equiv M\left(\frac{I_{1}}{I_{2}}\right), N \equiv N\left(\frac{I_{1}}{I_{2}}\right)$ then the equations (1)-(2) and (4) are invariant with respect to the conformal group. It is well known that conformal symmetry of Maxwell equations in vacuum was discovered by Bateman and Cunmigham (see [1,2]). Surprisingly but true the symmetry for the electromagnetic fields in moving media has not been investigated at all. In this paper we study the symmetry properties of Maxwell equations (1)-(2) together with additional constitutive equations in moving medium. 


\section{Classical Symmetry of Differential Equations for Electromagnetic Field}

Let consider the system of Maxwell equations (1)-(2) together with the Minkowski constitutive equations in the following form

$$
\vec{D}+\vec{u} \times \vec{H}=\varepsilon(\vec{E}+\vec{u} \times \vec{B}), \quad \vec{B}+\vec{E} \times \vec{u}=\mu(\vec{H}+\vec{D} \times \vec{u})
$$

where $\vec{u}$ is the velocity of the medium, $\varepsilon$ is the permittivity and $\mu$ is the permeance of stationary medium.

The following theorems have been proved.

Theorem 1. The system of equations (1)-(2) is invariant with respect to infinitedimensional Lie algebra whose basic elements are given by

$$
Q=X+\eta^{j^{a}} \frac{\partial}{\partial j^{a}}+\eta^{\rho^{a}} \frac{\partial}{\partial \rho}
$$

where

$$
\eta^{j^{a}}=-d j^{a}+\xi_{b}^{a} j^{b}+\xi_{0}^{a} \rho, \eta^{\rho}=-d \rho+\xi_{0}^{0} \rho+\xi_{b}^{0} j^{b}, d=-\left(\xi_{0}^{0}+\xi_{1}^{1}+\xi_{2}^{2}+\xi_{3}^{3}\right) .
$$

Proof: The proof of theorem requires long cumbersome calculations which are omitted here. We use in principle the standard Lie scheme which is reduced to realization of the following algorithm:

Step 1. The prolongation of infinitesimal operator $Q$ is constructed by using Lie formulae [8].

Step 2. Using the infinitesimal invariance condition [8]

$$
\left.\underset{1}{Q L \Psi}\right|_{L \Psi=0}=0
$$

where $Q$ is the first prolongation of operator $Q$ and $L \Psi=0$ is the system of equations (1)-(2) (symbolic form) we obtain the corresponding determining equations for the functions $\eta^{\rho}$, and $\eta^{j^{a}}$.

Step 3. Solving the corresponding determining equations we obtain the conclusion of the theorem.

From the invariance condition (8) for the equation (1) we obtain $\eta^{j^{a}}=-d j^{a}+$ $\xi_{b}^{a} j^{b}+\xi_{0}^{a} \rho$. By applying the criterium (8) we have $\eta^{\rho}=-d \rho+\xi_{0}^{0} \rho+\xi_{b}^{0} j^{b}$. As follows from [4] the invariance condition for the equations (2) gives no restriction on $\eta^{j^{a}}$ and $\eta^{\rho}$. 
Theorem 2. System of equation (1)-(2), (5) is invariant with respect to conformal Lie group with generators

$$
\begin{aligned}
& P_{0}=\partial_{t} ; \quad P_{a}=\partial_{x_{a}} \\
& J_{a b}=x_{a} \partial_{x_{b}}-x_{b} \partial_{x_{a}}+S_{a b}+V_{a b}+R_{a b} \\
& J_{0 a}=x_{0} \partial_{x_{a}}-x_{a} \partial_{x_{0}}+S_{o a}+V_{0 a}+R_{0 a} \\
& D=t \partial_{t}-x_{a} \partial_{x_{a}}-2\left(E_{k} \partial_{E_{k}}+B_{k} \partial_{B_{k}}+D_{k} \partial_{D_{k}}+H_{k} \partial_{H_{k}}\right)-3\left(j_{k} \partial_{j_{k}}-\rho \partial_{\rho}\right) \\
& K_{\mu}=2 x_{\mu} D-x^{2} P_{\mu}+2 x^{k}\left(S_{\mu k}+V_{\mu k}+R_{\mu k}\right), \quad \mu, k=0,1,2,3 \\
& S_{a b}=E_{a} \partial_{E_{b}}-E_{b} \partial_{E_{a}}+B_{a} \partial_{B_{b}}-B_{b} \partial_{B_{a}}+D_{a} \partial_{D_{b}}-D_{b} \partial_{D_{a}}+H_{a} \partial_{H_{b}}-H_{b} \partial_{H_{a}} \\
& S_{0 a}=\epsilon_{a b c}\left(E_{b} \partial_{B_{c}}-B_{b} \partial_{E_{c}}+D_{b} \partial_{H_{c}}-H_{b} \partial_{D_{c}}\right) \\
& V_{a b}=u_{a} \partial_{u_{b}}-u_{b} \partial_{u_{a}}, \quad V_{0 a}=\partial_{u_{a}}-u_{a}\left(u_{b} \partial_{u_{b}}\right) \\
& R_{a b}=j_{a} \partial_{j_{b}}-j_{b} \partial_{j_{a}}, \quad R_{0 a}=j_{a} \partial_{\rho}+\rho \partial_{j_{a}} .
\end{aligned}
$$

To prove the theorem we use the standard Lie scheme and therefore it is given without proof.

As follows from the theorem vectors $\vec{D}, \vec{B}, \vec{E}, \vec{H}$ are transformed under a linear representation of invariance group and the velocity of moving medium is transformed nonlinearly. The components of the velocity $\vec{u}$ are transformed in the following way

$$
u_{k} \rightarrow u_{k}^{\prime}=\frac{u_{k} \sigma-2 b_{0} x_{k}-2 b_{0}^{2} x_{k}\left(x_{0}-\vec{x} \cdot \vec{u}\right)}{1+2 b_{0}\left(x_{0}-\vec{x} \cdot \vec{u}\right)+b_{0}^{2}\left(x_{0}^{2}+\vec{x}^{2}-2 x_{0} \vec{x} \cdot \vec{u}\right)}
$$

where $b_{0}$ is the group parameter under the transformations generated by $K_{0}$ and $\sigma=1+2 b_{0} x_{0}+b_{0}^{2}\left(x_{0}^{2}-\vec{x}^{2}\right)$. Operators $K_{a}$ generate the following transformations for the velocity vector

$$
\begin{aligned}
& u_{a} \rightarrow u_{a}^{\prime}=\frac{u_{a} \delta+2\left(x_{0}-\vec{x} \cdot \vec{u}\right)\left(b_{a}-b_{a}^{2} x_{a}\right)-2 b_{a} u_{a}\left(x_{a}+b_{a} x^{2}\right)}{\delta+2 b_{a} x_{0}\left(x_{0}-\vec{x} \cdot \vec{u}\right)-2 b_{a} u_{a} x_{0}} \\
& u_{c} \rightarrow u_{c}^{\prime}=\frac{u_{c} \delta+2\left(x_{0}-\vec{x} \cdot \vec{u}\right) b_{a}^{2} x_{c}-2 b_{a} u_{a} x_{c}}{\delta+2 b_{a}^{2} x_{0}\left(x_{0}-\vec{x} \cdot \vec{u}\right)-2 b_{a} u_{a} x_{0}}, \quad c \neq a
\end{aligned}
$$

where $\delta=1-2 b_{a} x_{a}-b_{a}^{2} x^{2}, x^{2}=x_{0}^{2}-\vec{x}^{2}, b_{a}$ are the group parameters and there is no summation over $a$. 
Remark 3. If the permittivity $\epsilon$ and the permeance $\mu$ are functions of the ratio of $\vec{B}^{2}-\vec{E}^{2}$ and $\vec{B} \cdot \vec{E}$

$$
\epsilon=\epsilon\left(\frac{\vec{B}^{2}-\vec{E}^{2}}{\vec{B} \cdot \vec{E}}\right), \quad \mu=\mu\left(\frac{\vec{B}^{2}-\vec{E}^{2}}{\vec{B} \cdot \vec{E}}\right)
$$

then the nonlinear system of (1)-(2), (5) and (13) is invariant with respect to the Lie group of conformal transformations of dependent and independent variables. Thus the system of Maxwell equations (1)-(2), (5) in a moving external medium is invariant with respect to conformal group. And here, the velocity is changed nonlinearly under the transformations generated by $K_{\mu}$ according to formulae (10), (11) and (12).

In the all above given equations the fields $\vec{D}, \vec{B}, \vec{E}, \vec{H}$ are transformed in a linear way. Here, we give one more example of nonlinear system which is also conformally invariant but vector fields $\vec{E}, \vec{H}$ are transformed in a nonlinear way. The system has the form

$$
\frac{\partial \Sigma_{k}}{\partial x_{0}}+\Sigma_{l} \frac{\partial \Sigma_{k}}{\partial x_{l}}=0, \quad k, l=1,2,3
$$

where $\Sigma_{k}=E_{k}+\mathrm{i} H_{k}$. The complex system (14) is equivalent to the real system of equations for $\vec{E}$ and $\vec{H}$

$$
\frac{\partial E_{k}}{\partial x_{0}}+E_{l} \frac{\partial E_{k}}{\partial x_{l}}-H_{l} \frac{\partial H_{k}}{\partial x_{l}}=0, \quad \frac{\partial H_{k}}{\partial x_{0}}-H_{l} \frac{\partial E_{k}}{\partial x_{l}}+E_{l} \frac{\partial H_{k}}{\partial x_{l}}=0 .
$$

By using the Lie algorithm [8] we have proved the following theorem.

Theorem 4. The system of equation (15) is invariant with respect to 24-dimensional Lie algebra with generators

$$
\begin{aligned}
P_{\mu} & =\partial x_{\mu} \\
J_{k l}^{(1)} & =x_{k} \partial_{x_{l}}-x_{l} \partial_{x_{k}}+E_{k} \partial_{E_{l}}-E_{l} \partial_{E_{k}}+H_{k} \partial_{H_{l}}-H_{l} \partial_{H_{k}} \\
J_{k l}^{(2)} & =x_{k} \partial_{x_{l}}+x_{l} \partial_{x_{k}}+E_{k} \partial_{E_{l}}+E_{l} \partial_{E_{k}}+H_{k} \partial_{H_{l}}+H_{l} \partial_{H_{k}} \\
G_{a}^{(1)} & =x_{0} \partial_{x_{a}}+\partial_{E_{a}} \\
G_{a}^{(2)} & =x_{a} \partial_{x_{0}}-\left(E_{a} E_{k}-H_{a} H_{k}\right) \partial_{E_{a}}-\left(E_{a} H_{k}+H_{a} E_{k}\right) \partial_{H_{k}}
\end{aligned}
$$




$$
\begin{aligned}
D_{0}= & x_{0} \partial_{x_{0}}-E_{k} \partial_{E_{k}}-H_{k} \partial_{H_{k}} \\
D_{a}= & \left.x_{a} \partial_{x_{a}}+E_{k} \partial_{E_{k}}+H_{k} \partial_{H_{k}} \quad \text { (no summation over } a\right) \\
K_{0}= & x_{0}^{2} \partial_{x_{0}}+x_{0} x_{k} \partial_{x_{k}}+\left(x_{k}-x_{0} E_{k}\right) \partial_{E_{k}}-x_{0} H_{k} \partial_{H_{k}} \\
K_{a}= & x_{0} x_{a} \partial_{x_{0}}+x_{a} x_{k} \partial_{x_{k}}+\left[x_{k} E_{a}-x_{0}\left(E_{a} E_{k}-H_{a} H_{k}\right)\right] \partial_{E_{k}} \\
& +\left[x_{k} H_{a}-x_{0}\left(H_{a} E_{k}-E_{a} H_{k}\right)\right] \partial_{H_{k}} .
\end{aligned}
$$

The invariance algebra of the system (15) given by (16) contains Poincare , conformal, and Galilei algebras as subalgebras. The operators $J_{0 k}=G_{k}^{1}+G_{k}^{(2)}$ generate the standard transformations for $x$

$$
\begin{aligned}
x_{0}^{\prime} & =x_{0} \cosh \theta_{k}+x_{k} \sinh \theta_{k} \\
x_{k}^{\prime} & =x_{k} \cosh \theta_{k}+x_{0} \sinh \theta_{k} \\
x_{l}^{\prime} & =x_{l} \quad \text { if } l \neq k
\end{aligned}
$$

and nonlinear transformations for $\vec{E}, \vec{H}$

$$
\begin{aligned}
\vec{E}_{k}^{\prime}+\mathrm{i} \vec{H}_{k}^{\prime} & =\frac{\left(E_{k}+\mathrm{i} H_{k}\right) \cosh \theta_{k}+\sinh \theta_{k}}{\left(E_{k}+\mathrm{i} H_{k}\right) \sinh \theta_{k}+\cosh \theta_{k}} \\
\vec{E}_{k}^{\prime}-\mathrm{i} \vec{H}_{k}^{\prime} & =\frac{\left(E_{k}-\mathrm{i} H_{k}\right) \cosh \theta_{k}+\sinh \theta_{k}}{\left(E_{k}-\mathrm{i} H_{k}\right) \sinh \theta_{k}+\cosh \theta_{k}} \\
\vec{E}_{l}^{\prime}+\mathrm{i} \vec{H}_{l}^{\prime} & =\frac{E_{l}+\mathrm{i} H_{l}}{\left(E_{k}+\mathrm{i} H_{k}\right) \sinh \theta_{k}+\cosh \theta_{k}}, \quad l \neq k \\
\vec{E}_{l}^{\prime}-\mathrm{i} \vec{H}_{l}^{\prime} & =\frac{E_{l}-\mathrm{i} H_{l}}{\left(E_{k}-\mathrm{i} H_{k}\right) \sinh \theta_{k}+\cosh \theta_{k}}, \quad l \neq k .
\end{aligned}
$$

Thus we conclude that the system of Maxwell equation (1)-(2) and constitutive Minkowski equations (5) are conformally invariant just the same as the linear Maxwell equations for electromagnetic fields in vacuum. This symmetry can be successfully used for construction solutions of Maxwell equations in moving media by method of comparison of electrodynamic systems [7].

\section{References}

[1] Bateman H., The Transformations of the Electrodynamical Equations, Proc. London Math. Soc. 8 (1909) 223-264.

[2] Cunningham E., The Principle of Relativity in Electrodynamics and Extention There of., Proc. London Math. Soc. 8 (1909) 77-98. 
[3] Fushchych W. and Nikitin A., Symmetries of Maxwell's Equation, Dordrecht, Reidel, 1994.

[4] Fushchych W. and Tsifra I., On the Symmetry of Nonlinear Electrodynamic Equations, Teoret. Mat. Fiz. 64 (1985) 41-50.

[5] Goldin G. and Shtelen V., On Galilean Invariance and Nonlinearity in Electrodynamics and Quantum Mechanics, Phys. Lett. A279 (2001) 321-326.

[6] Goldin G. and Shtelen V., Generalization of Yang-Mills Theory with Nonlinear Constitutive Equations, J. Phys. A: Math. Gen. 37 (2004) 10711-10718.

[7] Miller M., Sorokin J. and Stepanov N., Covariance of Maxwell Equation and Comparison of Electrodynamic Systems, Uspechi Fiz. Nauk 121 (1977) 525-537.

[8] Olver P., Applications of Lie Groups to Differential Equations, Springer, New York, 1986

Ivan Tsyfra

Institute of Mathematics

University of Bialystok

Bialystok 15-267

POLAND

E-mail address: tsyfra@math.uwb.edu.pl 